POS PROCEEDINGS

\title{
New Limits on WIMP Dark Matter from Annual Modulation Analysis of the CDEX Experiment at the China Jinping Underground Laboratory
}

\author{
Hau-Bin Li (on behalf of CDEX Collaboration) ${ }^{a, *}$ \\ ${ }^{a}$ Institute of Physics, Academia Sinica, Taipei \\ E-mail: lihb@gate.sinica.edu.tw
}

\begin{abstract}
We present results on light weakly interacting massive particle (WIMP) searches [1] with annual modulation (AM) analysis on data from a 1-kg mass p-type point-contact germanium detector of the CDEX-1B experiment at the China Jinping Underground Laboratory. The 90\% C.L. allowed regions implied by the DAMA/LIBRA and CoGeNT AM-based analysis are excluded at $>99.99 \%$ and $>98 \%$ C.L., respectively. These results correspond to the best sensitivities at $m_{\chi}<6 \mathrm{GeV} / \mathrm{c}^{2}$ among WIMP AM measurements.
\end{abstract}

40th International Conference on High Energy physics - ICHEP2020

July 28 - August 6, 2020

Prague, Czech Republic (virtual meeting)

\footnotetext{
${ }^{*}$ Speaker
} 
The Weakly Interacting Massive Particle (WIMP, denoted as $\chi$ ) is a favored candidate of dark matter (DM). The direct laboratory searches of WIMPs can be probed by WIMP-nucleus $(\chi-\mathrm{N})$ elastic scattering. Within the astrophysical dark matter halo model, the $\chi$-N rates have distinctive annual modulation (AM) feature with maximum intensity at June and a period of $1 \mathrm{yr}$ due to the Earth's motion relative to to the galaxy dark matter distribution. AM analysis of $\chi$ - $\mathrm{N}$ can provide smoking-gun signatures for WIMPs independent of background modeling.

Positive results were concluded at significance of $12.9 \sigma$ and $2.2 \sigma$ from AM-based analysis of DAMA/LIBRA [2, 3] and CoGeNT [4] experiments, respectively. However, these interpretations were challenged by integrated rate experiments with liquid xenon [5-7], cryogenic bolometer [810], ionization germanium [11] detectors and were probed and excluded by AM analysis from XMASS-1 [12] and XENON100 [13, 14] experiments as well.

The CDEX experiments, located in the China Jinping Underground Laboratory (CJPL) with about $2400 \mathrm{~m}$ of rock overburden, utilizes $p$-type point contact germanium detectors ( $p \mathrm{PCGe}$ ) [15] for dark matter direct detection. The low analysis threshold of about 200 eVee implies AM studies with germanium can complement the liquid xenon results. It provides an alternative probe to the allowed parameter space of DAMA/LIBRA and CoGeNT and extends the reach of AM test to lower lower $m_{\chi}$.

The CDEX-1B experiment is the second phase of the CDEX experiment and has previously given the upper limits for spin-independent (SI) and spin-dependent (SD) cross sections by the $\chi$-N recoil spectral analysis [16]. The $p$ PCGe target of mass $1 \mathrm{~kg}$ (fiducial mass of $939 \mathrm{~g}$, after corrections due to a $0.88 \pm 0.12 \mathrm{~mm}$ surface layer) was shielded with $20 \mathrm{~cm}$ of copper, $20 \mathrm{~cm}$ of polyethylene and $20 \mathrm{~cm}$ of lead, from inside to outside. The whole setup was assembled inside a $6 \mathrm{~m}(H) \times 8 \mathrm{~m}(L) \times 4 \mathrm{~m}(W)$ polyethylene room with wall thickness of $1 \mathrm{~m}$. The target was enclosed by an $\mathrm{NaI}(\mathrm{Tl})$ anti-Compton detector from September 27, 2014 to August 2, 2017 (Run-1), and subsequently without $\mathrm{NaI}(\mathrm{Tl})$ (replaced by passive copper shielding) from August 4, 2017 till December 2, 2018 (Run-2). The gaps from December 27, 2014 to March 8, 2015 and from March 16, 2016 to June 02,2016 were due to calibration with neutron gamma-ray sources, respectively. The two runs have 751.3 and 428.1 live days, respectively, and together span a total of 1527 calendar days ( $\sim 4.2 \mathrm{yr})$, with the total exposure of $1107.5 \mathrm{~kg}$-day.

The $4.2 \mathrm{yr}$ of CDEX-1B data are separated into 35 subdatasets in different time bins, each with about 1 month of live time. WIMP candidate events in the bulk of the detector are selected via some basic cuts and the bulk or surface $(\mathrm{B} / \mathrm{S})$ events discrimination. The $\mathrm{B} / \mathrm{S}$ correction procedure is done by likelihood fitting of the bulk or surface rise-time distribution probability density functions (PDFs) and has no cut efficiency associated [16].

Stability of $\chi$-N candidate events with time is demonstrated in Fig. 1 with the bulk event count rates after $\mathrm{B} / \mathrm{S}$ correction at three energy ranges which are most relevant to the sensitivities at $m_{\chi}=8 \mathrm{GeV} / \mathrm{c}^{2}$.

The only requirement for AM analysis is to have stable background with time. The modeling of their origins and spectral shapes, which are sources of uncertainties in the time-integrated spectral analysis, is not involved. We adopted a scenario of the time-independent background contribution plus an exponentially time-dependent background contribution from the L-shell x-rays from cosmogenic isotopes. The expected time dependence due to the cosmogenic origin background contributions was observed. It dominated the background in energy ranges of 1.0-1.4 keVee. 


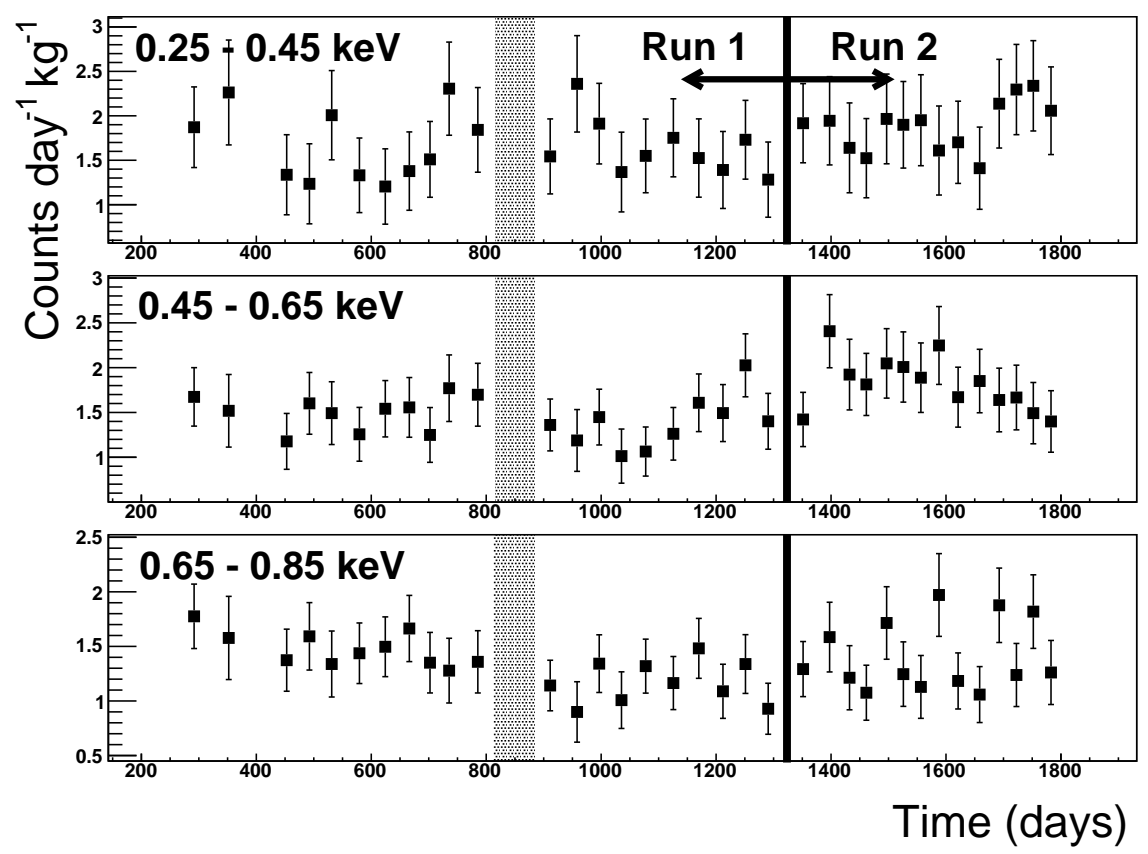

Figure 1: The B/S corrected bulk event counts versus time at three energy ranges, where the shaded area denotes the period of gamma source calibration. A bin size of $200 \mathrm{eVee}$ is used in this plot, while different bin size is adopted in the analysis.

The time-independent background levels of every energy bin were taken as free parameters and were uncorrelated between Run-1 and Run- 2 due to the different shielding configurations. The unmodulated $\chi-\mathrm{N}$ rates were treated as a component of the constant background in AM analysis.

Data at $0.25-5.8$ keVee were analyzed, below the region of internal K-shell X-rays. The selected energy-bin-sizes are 50 eVee, 100 eVee and 200 eVee for measured energy at $<0.8$ keVee, $0.8-1.6$ keVee and $>1.8 \mathrm{keVee}$, respectively, according to the requirements of statistical accuracy in $\mathrm{B} / \mathrm{S}$ correction.

For each of $i$ th energy bin, a minimum $\chi^{2}$ analysis was performed simultaneously, with

$$
\chi_{i}^{2}=\sum_{k \in \text { Run }}^{2} \sum_{j \in \text { Time }}^{N} \frac{\left(n_{i j k}-P_{i j k}-B_{i k}-A_{i} \cos \left(\frac{2 \pi\left(t_{j}-\phi\right)}{T_{y r}}\right)\right)^{2}}{\Delta_{i j k}^{2}},
$$

where $\phi$ (fixed at 152.5 days) and $T_{y r}$ (fixed at 1 year) are, respectively, the modulation phase and period. The corrected counts of bulk events are denoted by $n_{i j k}$ corresponding to the respective bin with $i, j, k=$ (energy, time, run). $P_{i j k}$ is the time-varying background contributions of the L-shell $\mathrm{X}$-rays from long-lived isotopes such as ${ }^{68} \mathrm{Ge},{ }^{68} \mathrm{Ga}$ and ${ }^{65} \mathrm{Zn}$, with intensities fixed by the measured $\mathrm{K}$-shell X-rays at $8.5-10.8 \mathrm{keV}, B_{i k}$ is the background level, in which we adopted a time-independent background scenario, and $\Delta_{i j k}{ }^{2}$ are the combined statistical and systematic errors dominated by the $\mathrm{B} / \mathrm{S}$ correction [16]. There are in total 40 energy bins, with 35 time bins divided into 2 runs.

The data are first studied with a model-independent analysis without invoking astrophysical models and parameters. The modulation amplitude $A_{i}$ of individual energy bins are treated inde- 


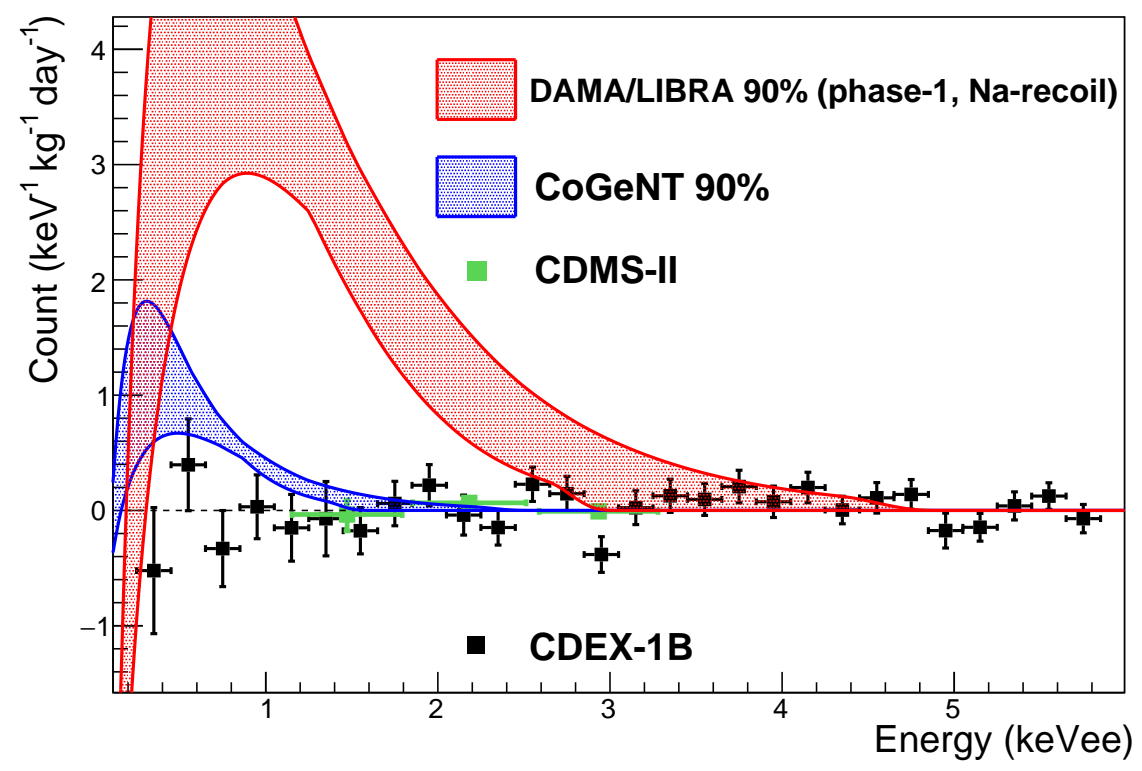

Figure 2: Best-fit solutions of modulation amplitude $A_{i}$. The distributions show consistency with null results, i.e., no significant modulation signatures. For illustration purpose, we adopted 200 eVee bin-size for all energy ranges. Derived modulation signals from 90\% C.L. allowed region of CoGeNT [4, 17], DAMA/LIBRA (Na-recoil) [18] and best-fit modulation amplitudes of CDMS-II distributed results [19] are superimposed. A bin size of 200 eVee is used in this plot, while different bin size is adopted in the analysis.

pendently, from which the best-fit results $\chi^{2} /$ d.o.f $=\sum \chi_{i}^{2} /$ d.o.f $=1280.47 / 1280$ are shown in Fig. 2. The distribution of $A_{i}$ is consistent with null results, showing no evidence of modulation behavior. The null hypothesis test gave a $\chi^{2} /$ d.o.f $=1330.27 / 1320$. The difference in $\chi^{2}$ between null hypothesis and independent-amplitude analysis is within $\chi^{2}$ distribution at $p$ value $=0.14$.

For the model-dependent analysis, the individual $A_{i}$ are correlated with a known function of $m_{\chi}, \sigma_{S I}, E$ and bin-size, while the function is related to the applied astrophysics models. Data are then analyzed under the standard spherical isothermal galactic halo model, with a most probable speed of $v_{0}=220 \mathrm{~km} / \mathrm{s}$, a galactic escape velocity of $v_{\text {esc }}=544 \mathrm{~km} / \mathrm{s}$, an Earth's velocity related to dark matter of $v_{E}=\left\{232+15 \cos \left(2 \pi(t-\phi) / T_{y r}\right)\right\} \mathrm{km} / \mathrm{s}$ and local dark matter density of $0.3 \mathrm{GeV} /\left(\mathrm{c}^{2} \mathrm{~cm}^{3}\right)$. Quenching factor of Ge is derived by the TRIM software package [20] with a $10 \%$ systematic error adopted for the analysis. Best-fit values of unconstrained $\sigma_{S I}$ are then valuated by minimizing $\sum \chi_{i}^{2}$ of Eq. (1), The Unified Approach [21] is then used to place the upper bounds of positive definite $\sigma_{S I}$ at different $m_{\chi}$.

The best-fit solution at $m_{\chi}=7.9 \mathrm{GeV} / \mathrm{c}^{2}$ is $\sigma_{S I}=(-0.37 \pm 1.43) \times 10^{-41} \mathrm{~cm}^{2}\left(\chi^{2}=1330.20 / 1319\right)$, or equivalently, $\sigma_{S I}<1.99 \times 10^{-41} \mathrm{~cm}^{2}$ at $90 \%$ C.L. The upper limits at $90 \%$ C.L. on $\sigma_{S I}$ are derived and shown in Fig. 3. The results refute the 90\% C.L. allowed regions inferred from AM-based analysis of DAMA/LIBRA-phase 1 low- $m_{\chi}$ (Na-recoil) $[2,3,18]$ and CoGeNT [4] experiments, providing an exclusion at $>99.99 \%$ and $>98 \%$ C.L., respectively. The DAMA/LIBRA high- $m_{\chi}$ region (I-recoil) is not probed in this analysis.

The $\mathrm{B} / \mathrm{S}$ discrimination contributes less than $8 \%$ deviation of $\sigma_{S I}$, and the uncertainty of K/L 
ratios is also incorporated in the systematic uncertainty budget.

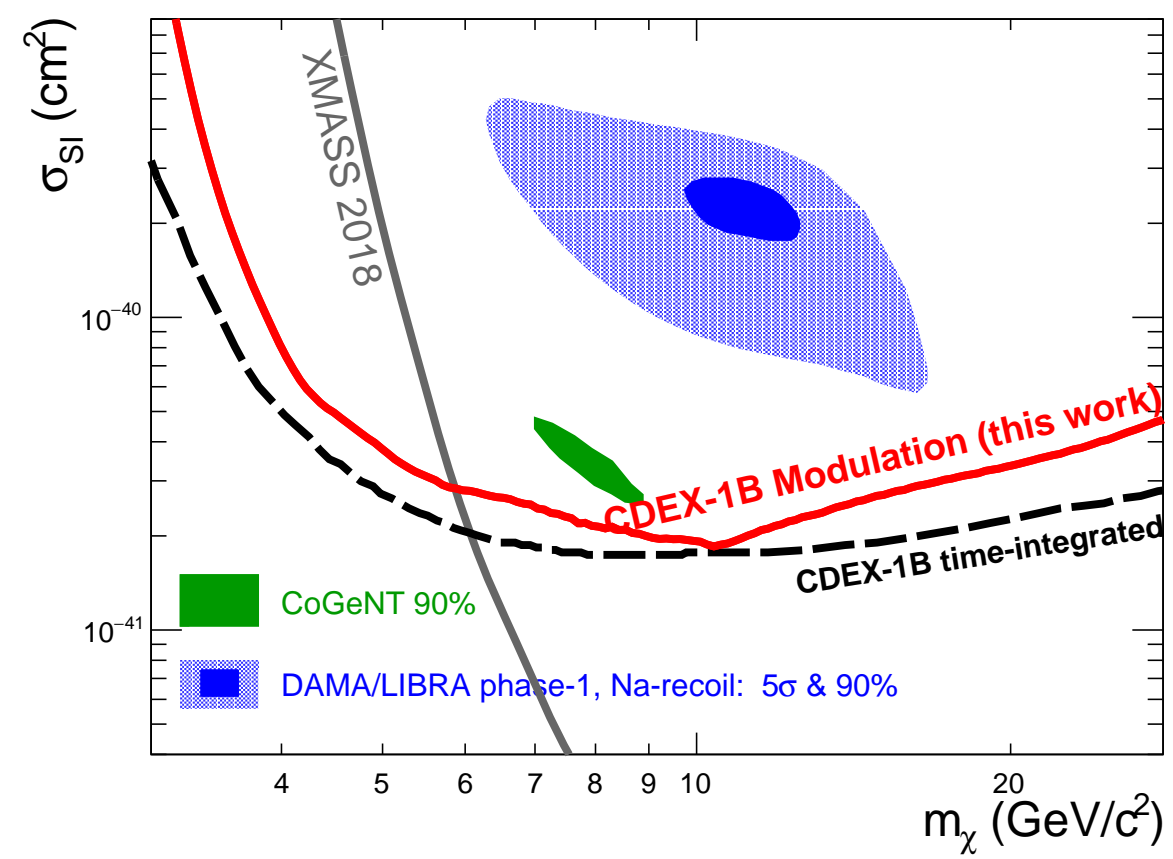

Figure 3: Limits at 90\% C.L. from CDEX-1B AM-analysis (red) on spin-independent WIMP-nucleon cross section. Also shown are other AM-based results: 90\% C.L. upper limits of XMASS-1 (dark-gray) [12], allowed regions of DAMA/LIBRA (Na-recoil, pale blue: $5 \sigma$, blue: $90 \%$ C.L.) $[2,3,18]$ and CoGeNT (green: 90\% C.L.) [4]. Constraints from the CDEX-1B time-integrated spectral analysis [16] are displayed (black dotted line) as comparison.

\section{References}

[1] L.T. Yang et al., Search for light weakly-interacting-massive-particle dark matter by annual modulation analysis with a point-contact germanium detector at the China Jinping Underground Laboratory, Phys. Rev. Lett. 123 (2019) 221301.

[2] R. Bernabei et al., New results from DAMA/LIBRA, Eur. Phys. J. C 67 (2010) 39.

[3] R. Bernabei et al., Final model independent result of DAMA/LIBRA-phase1, Eur. Phys. J. C 73 (2013) 2648.

[4] C.E. Aalseth et al., CoGeNT: A search for low-mass dark matter using p-type point contact germanium detectors, Phys. Rev. D 88 (2013) 012002.

[5] E. Aprile et al., Dark matter search results from a one ton-year exposure of XENONIT, Phys. Rev. Lett. 121 (2018) 111302.

[6] D.S. Akerib et al., Results from a search for dark matter in the complete LUX exposure, Phys. Rev. Lett. 118 (2017) 021303. 
[7] X. Cui et al., Dark matter results from 54-ton-day exposure of PandaX-II experiment, Phys. Rev. Lett. 119 (2017) 181302.

[8] R. Agnese et al., Search for low-mass weakly interacting massive particles with SuperCDMS, Phys. Rev. Lett. 112 (2014) 241302.

[9] R. Agnese et al., New results from the search for low-mass weakly interacting massive particles with the CDMS low ionization threshold experiment, Phys. Rev. Lett. 116 (2016) 071301.

[10] G. Angloher et al., Results on light dark matter particles with a low-threshold CRESST-II detector, Eur. Phys. J. C 76 (2016) 25.

[11] W. Zhao et al., Search of low-mass WIMPs with a p-type point contact germanium detector in the CDEX-1 experiment, Phys. Rev. D 93 (2016) 092003.

[12] K. Abe et al., Direct dark matter search by annual modulation with 2.7 years of XMASS-I data, Phys. Rev. D 97 (2018) 102006.

[13] E. Aprile et al., Search for event rate modulation in XENON100 electronic recoil data, Phys. Rev. Lett. 115 (2015) 091302.

[14] E. Aprile et al., Search for electronic recoil event rate modulation with 4 years of XENON100 data, Phys. Rev. Lett. 118 (2017) 101101.

[15] P.N. Luke et al., Low capacitance large volume shaped-field germanium detector, IEEE Trans. Nucl. Sci. 36 (1989) 926.

[16] L.T. Yang et al., Limits on light WIMPs with a $1 \mathrm{~kg}$-scale germanium detector at 160 evee physics threshold at the China Jinping Underground Laboratory, Chin. Phys. C 42 (2018) 023002 .

[17] C.E. Aalseth et al., Search for an annual modulation in three years of CoGeNT dark matter detector data, arXiv: 1401.3295.

[18] C. Savage et al., Compatibility of DAMA/LIBRA dark matter detection with other searches, J. Cosmol. Astropart. Phys. 04 (2009) 010.

[19] Z. Ahmed et al., Search for annual modulation in low-energy CDMS-II data, arXiv: 1203.1309.

[20] J.F. Ziegler et al., SRIM-2003, Nucl. Instr. Meth. Phys. Res. B 219 (2004) 1027.

[21] G.J. Feldman and R.D. Cousins, Unified approach to the classical statistical analysis of small signals, Phys. Rev. D 57 (1998) 3873. 